\title{
Latent membrane protein 1 mediates the resistance of nasopharyngeal carcinoma cells to TRAIL-induced apoptosis by activation of the PI3K/Akt signaling pathway
}

\author{
SHI-SHENG LI, SHU YANG, SHUANG WANG, XIN-MING YANG, QING-LAI TANG and SHU-HUI WANG \\ Department of Otolaryngology, Head and Neck Surgery, The Second Xiangya Hospital, \\ Central South University, Changsha 410011, Hunan, P.R. China
}

Received June 24, 2011; Accepted July 25, 2011

DOI: 10.3892/or.2011.1423

\begin{abstract}
The 5-year survival rate of nasopharyngeal carcinoma (NPC) is still disappointing despite the much improved technologies in its treatment. Tumor necrosis factor-related apoptosis inducing ligand (TRAIL) can selectively induce apoptosis in most tumor cells while sparing normal cells. However, its potential in the treatment of NPC has been limited by the eventual emergence of drug resistance. Latent membrane protein 1 (LMP1) is a major oncogene of the human DNA tumor virus Epstein-Barr virus (EBV) and is associated with the development of NPC and the emergence of chemoresistance in NPC. In this study, we investigated the potential role of LMP1 in TRAIL resistance in CNE-1 NPC cells. Results show that overexpression of LMP1 could induce TRAIL resistance in NPC cells without influencing death receptors. The LMP1-induced TRAIL resistance is associated with increased expression of FLIP and elevated cleavage of caspase- 8 without altering the TRAIL-mediated mitochondrial events and Bid cleavage. Knockdown of the FLIP gene with siRNA prevented the LMP1-induced TRAIL resistance. Furthermore, we found that overexpression of LMP1 activated Akt. Inhibition of Akt with LY294002 completely prevented the LMP1-induced FLIP expression and TRAIL resistance. Together, these results show that LMP1 can inhibit the TRAIL-mediated apoptosis through activation of PI3K/Akt and expression of FLIP in CNE-1 NPC cells, and may provide new methods to prevent and reverse drug resistance in NPC.
\end{abstract}

Correspondence to: Dr Xin-Ming Yang, Department of Otolaryngology, Head and Neck Surgery, The Second Xiangya Hospital, Central South University, Changsha 410011, Hunan, P.R. China

E-mail: x16y2003@yahoo.com.cn

Key words: nasopharyngeal carcinoma, tumor necrosis factorrelated apoptosis inducing ligand, latent membrane protein 1, resistance, Akt, FLIP

\section{Introduction}

Tumor necrosis factor (TNF)-related apoptosis inducing ligand (TRAIL) is a cytokine of the TNF family and capable of inducing apoptotic cell death in a variety of cancer cells without having significant effect on normal cells (1). TRAIL induces apoptosis by binding to their cognate receptors, TRAIL-R1 (death receptor 4, DR4) and TRAIL-R2 (DR5) (2). The Fas-associated death domain (FADD) and caspase- 8 are recruited to the DR-TRAIL complex to form a death-inducing signaling complex (DISC) (2). The apoptotic signal is then transmitted through the extrinsic and intrinsic pathways. The extrinsic pathway is mitochondrion-independent but the intrinsic pathway is mitochondrion-dependent (3). Signals from DISC are linked to mitochondria by Bid, which contains a homologous domain of the Bcl-2 protein family. Bid is cleaved by caspase- 8 and then translocates to mitochondria to activate Bcl-2 family members Bax and Bak, leading to mitochondrial depolarization (4). Apoptotic factors are consequently released from mitochondria into cytosol to activate caspase-3 through formation of apoptosome complex (4). Studies have shown that some cancer cells are resistant to the apoptotic effect of TRAIL, the so-called TRAIL resistance (5). However, TRAIL resistance can be overcome by combining TRAIL with different chemotherapeutic drugs (6), indicating that an approach by combining multiple targets can be utilized to prevent or reverse TRAIL resistance in cancer cells. Thus, studying the factors that cause TRAIL resistance may be an important way to find more effective therapies for cancer.

Nasopharyngeal carcinoma (NPC) has a distinct racial and geographical distribution. It is common in south-east Asia and southern China but is rare in most other parts of the world. Most patients with NPC are diagnosed at an advanced stage. Radiotherapy alone was the standard treatment for NPC at all stages until 1990 (8). Despite significant progress in methods of radiotherapy, the outcome with radiotherapy alone has been disappointing. The 5-year survival rate is between $34-52 \%$ (9). Thus, finding more effective treatment for NPC has become an urgent priority. Death receptors have been detected in specimens of head and neck tumors but not in surrounding normal tissues (10). These findings support the notion that the presence of death receptors on some head and 
neck cancer may make them more susceptible to the TRAILmediated apoptosis. Some have shown robust induction of apoptosis in NPC cell line TW02 by TRAIL (11) while others had difficulty in inducing apoptosis in some NPC cell lines by TRAIL (12). The reason for the discrepancy is currently unknown. Persistent Epstein-Barr virus (EBV) infection can cause malignant diseases such as nasopharyngeal carcinoma, Hodgkin's lymphoma and Burkitt's lymphoma (13). Latent membrane protein 1 (LMP1) is the primary oncogene of EBV (14). LMP1 seems to immortalize and transform cells by simultaneously controlling signaling pathways that block apoptosis and stimulate cellular proliferation like a growth factor (15). LMP1 has been shown to be able to induce chemoresistance in nasopharyngeal carcinoma (16) and activate the PI3K/Akt pathway to promote cell survival (17). Activation of PI3-K/Akt pathway can protect cells from the TRAIL-induced apoptosis (18), and blockade of the PI3-K/Akt signaling pathway sensitizes some cancer cells to the TRAIL-induced apoptosis (19). In this study, we tested the hypothesis that overexpression of LMP1 could induce TRAIL resistance in NPC cells through activation of PI3K/Akt pathway and investigated the associated molecular mechanisms.

\section{Materials and methods}

Cell lines and reagents. CNE-1 is an EBV negative low differentiated nasopharyngeal squamous carcinoma cell line established by Cancer Research Institute, Zhongshan Medical University. The stable CNE-1 cells that express LMP1 (CNE-1-LMP1) was established by Cancer Research Institute, Xiangya School of Medicine, Central South University. All cells were cultured in RPMI-1640 (Invitrogen, Carlsbad, USA) media supplemented with $10 \%$ fetal bovine serum (Hyclone, Logan, USA) and penicillin/streptomycin, and maintained in a humidified atmosphere of $5 \% \mathrm{CO}_{2}$ at $37^{\circ} \mathrm{C}$. All experiments were performed with cells in exponential growth.

MTT cell viability assay. Cell viability was determined by using 3-(4,5-dimethylthiazolyl)-2,5-diphenyltetrazolium bromide (MTT) assays. NPC cells (5,000 cells per well) were plated in 96-well plates. After the treatment with TRAIL $(0-100 \mathrm{ng} / \mathrm{ml})$ for the indicated time, cells were incubated for 2-3 h with $0.5 \mathrm{mg} / \mathrm{ml}$ of MTT (Sigma-Aldrich Co., St. Louis, MO, USA) and lysed with dimethyl sulfoxide (DMSO). The optical density (OD) of each well was measured at $570 \mathrm{~nm}$ with a microplate reader (survival rate $=\mathrm{OD}_{\text {treat }} / \mathrm{OD}_{\text {control }}$ ).

Apoptosis assessment by Annexin V/PI staining. The levels of apoptosis were determined by using Annexin V/PI staining kit. After different treatments, cells were harvested. Cell pellets were suspended in $500 \mu \mathrm{l}$ binding buffer $(10 \mathrm{mM}$ HEPES, $\mathrm{pH} 7.4,140 \mathrm{mM} \mathrm{NaCl}, 1 \mathrm{mM} \mathrm{MgCl}{ }_{2}, 5 \mathrm{mM} \mathrm{KCl}, 2.5 \mathrm{mM}$ $\left.\mathrm{CaCl}_{2}\right)$ at the density of $1 \times 10^{6} / \mathrm{ml}$. Samples were incubated with $1 \mu \mathrm{l}$ Annexin V-FITC and $5 \mu \mathrm{l}$ PI for $5 \mathrm{~min}$ at room temperature in the dark and then measured on a FACSort flow cytometer (Becton-Dickinson, USA). Annexin V-FITC and PI fluorescence were detected in the FL-1 (green) and FL-2 (red) channels respectively after correction to the spectral overlap between the two channels. Data were analyzed by using CellQuest software.
Flow cytometric analysis of TRAIL receptors. NPC cells $\left(1 \times 10^{6}\right.$ cells $)$ from the culture media were detached and pelleted (1000 r.p.m. for $10 \mathrm{~min}$ ) and suspended in $500 \mu \mathrm{l}$ of the collected media. Cells were then incubated with $5 \mu \mathrm{l}$ of anti-DR4 or anti-DR5 polyclonal goat antibody (1:100, SigmaAldrich Co.) for $1 \mathrm{~h}$. After washing with PBS, FITC-conjugated rabbit anti-goat polyclonal antibody (1:200, Sigma-Aldrich Co.) was added to the cell suspensions and incubated for $1 \mathrm{~h}$ on ice. After rinsing with PBS, samples were analyzed with a FACSort flow cytometer (Becton-Dickinson). Data were analyzed by using CellQuest software.

FLIP siRNA transfection. pSilencer 3.1-H1 linear Vector was purchased from Invitrogen Corp. Small interference RNA (siRNA) was synthesized by GenePharma, Shanghai, China. The sequences of the siRNA are as follows: for FLIP sense 5'-GGAGCAGGGACAAGUUACA-3' and antisense 5'-UUCUCCGAACGUGUCACGU-3'. Negative control vector that expresses a hairpin siRNA with limited homology to any known sequences in the human was provided in the vector kit. Plasmid DNA was purified with CsCl-ethidium bromide gradient centrifugation. The purified DNA was diluted to $1 \mathrm{~g} / \mathrm{l}$ and frozen at $-20^{\circ} \mathrm{C}$. The transfection of siRNA was performed using Lipofectamine 2000 (Invitrogen) according to the manufacturer's instructions. In brief, cells were seeded into wells of a 6 -well plate at $10^{5}$ cells $/ \mathrm{cm}^{2}$ and cultured for 24 h until $60-70 \%$ confluence. Then FLIP siRNA or negative siRNA plasmid $(4 \mu \mathrm{l})$ was diluted with $100 \mu$ l OPTI-MEM (Invitrogen) for $5 \mathrm{~min}$ at room temperature. During the incubation period, $5 \mu \mathrm{l}$ Lipofectamine 2000 (Invitrogen) was diluted in $100 \mu \mathrm{l}$ OPTI-MEM. These two mixtures were combined, mixed gently and incubated for $20 \mathrm{~min}$ at room temperature for complex formation. This $200 \mu \mathrm{l}$ siRNA-Lipofectamine 2000 mixture was then added to cells. The transfected cells were cultured for $48 \mathrm{~h}$ and then assayed.

Western blot analysis. Cells were washed with PBS and dissolved in lysis buffer $\left(20 \mathrm{mM} \mathrm{Na} 2 \mathrm{PO}_{4}, 150 \mathrm{mM} \mathrm{NaCl}, 1 \%\right.$ Triton $\mathrm{X}-100,1 \%$ aprotinin, $1 \mathrm{mM}$ phenylmethylsulfonyl fluoride, $100 \mathrm{mM} \mathrm{NaF}$ and $2 \mathrm{mM} \mathrm{Na}_{3} \mathrm{VO}_{4}$ ). Proteins were separated by polyacrylamide gel electrophoresis on a sodium dodecyl sulfate-polyacrylamide gel and transferred to a polyvinylidene difluoride membrane. Membranes were incubated with the first antibody overnight at $4^{\circ} \mathrm{C}$. The next day, the membranes were washed with phosphate-buffered saline (PBS) three times and then membranes were incubated with horseradish peroxidaseconjugated goat anti-rabbit secondary antibodies (all were obtained from Santa Cruz Biotechnology, Santa Cruz, CA). Finally, proteins were detected by the enhanced chemiluminescence (ECL) procedure.

Assessment of mitochondrial transmembrane potential. Assessment of mitochondrial transmembrane potential $(\Delta \Psi \mathrm{m})$ by the cationic lipophilic fluorochrome 3,3'-dihexyloxacarbocyanide iodide $\left[\mathrm{DiOC}_{6}(3)\right]$ (460 ng/ml, Molecular Probes, Eugene, OR, USA) was used to measure the mitochondrial transmembrane potential. Cells were treated for $24 \mathrm{~h}$ with TRAIL $(100 \mathrm{ng} / \mathrm{ml})$, then incubated for $30 \mathrm{~min}$ at $37^{\circ} \mathrm{C}$ in the media with $\mathrm{DiOC}_{6}$, washed twice with PBS and analysed in a FACSort flow cytometer. 

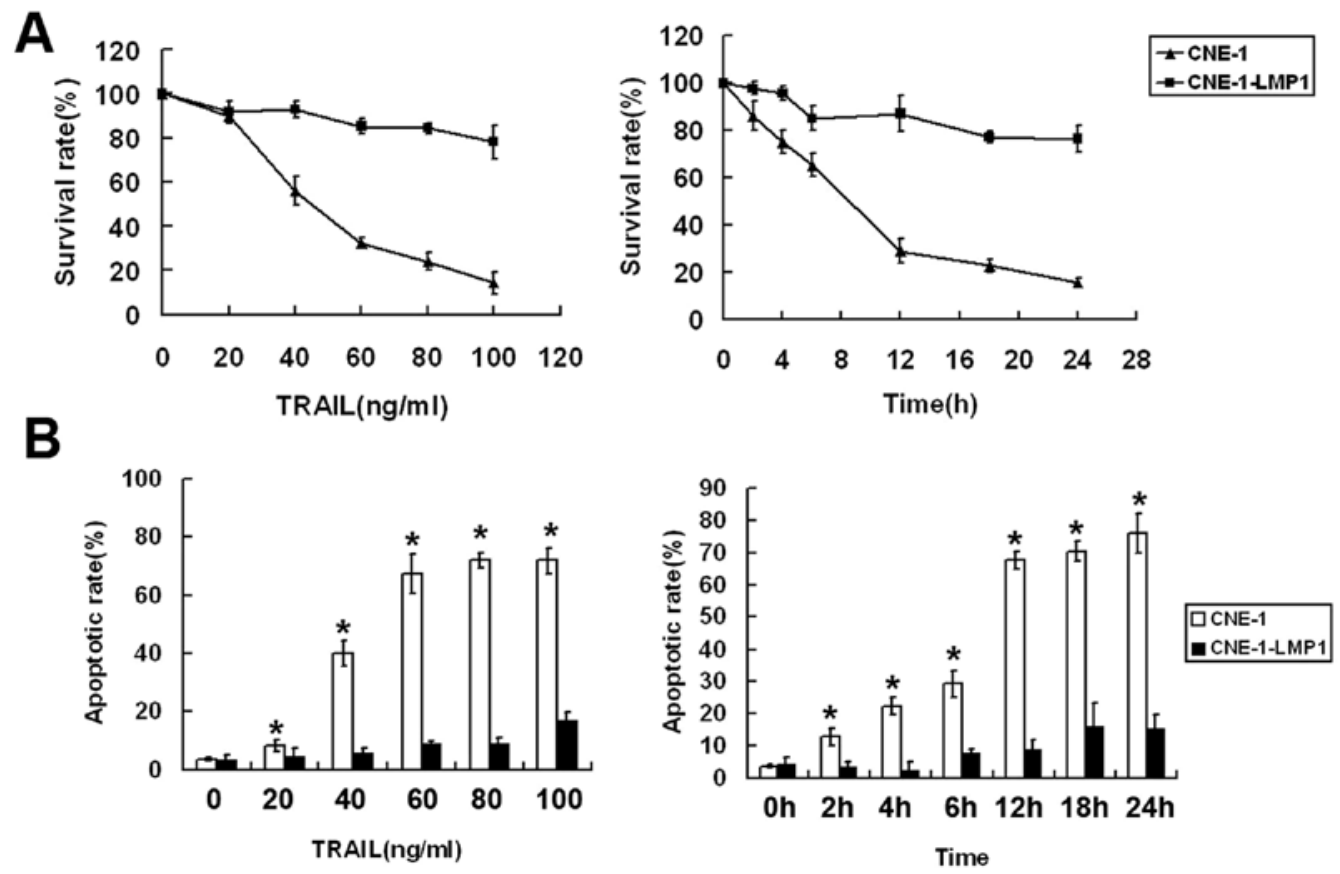

Figure 1. Overexpression of LMP1 protein regulates TRAIL resistance of NPC cell lines. (A) Cell viability of CNE-1 and CNE-1-LMP1 was detected by MTT assay after TRAIL treatment for $24 \mathrm{~h}$ or $100 \mathrm{ng} / \mathrm{ml}$ of TRAIL for different periods of time. Data are summarized as the mean \pm SD and represent at least three replicates. (B) Apoptosis was detected by Annexin V/PI assay after TRAIL treatment. The cell apoptotic rate was determined by flow cytometry analysis. Data were summarized as the mean \pm SD and represent at least three replicates. Differences between two groups were determined by the Student's $t$-test. ${ }^{*} P>0.05$ compared to CNE-1-LMP1 cells.

A

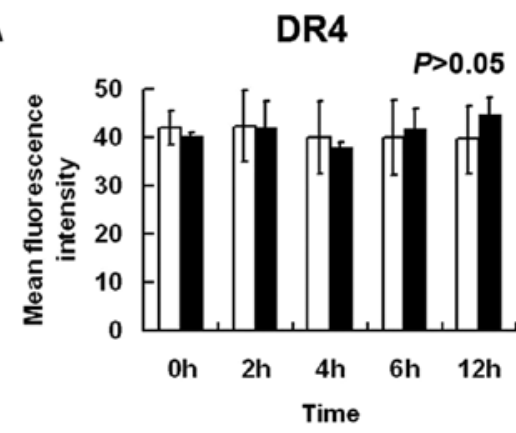

B

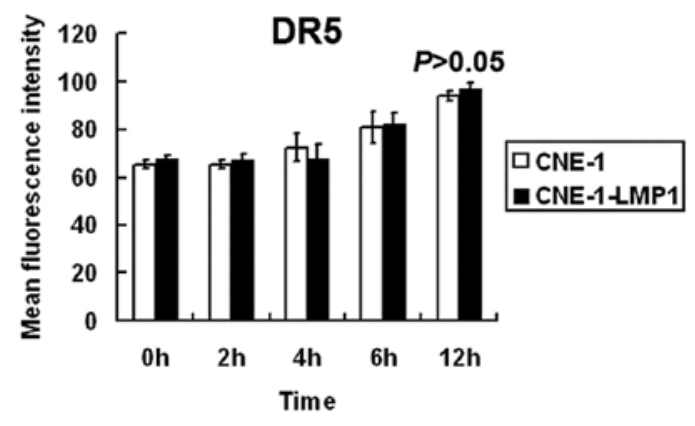

Figure 2. Surface expression of tumor necrosis factor-related apoptosis-inducing ligand (TRAIL) receptors (DR4 and DR5) was not increased in LMP1 overexpressive NPC cells. NPC cells were incubated with $100 \mathrm{ng} / \mathrm{ml} \mathrm{TRAIL}$ or solvent control for different periods of time and labeled on ice by indirect immunostaining for cell surface expression of TRAIL-receptors (DR4 and DR5). Non-specific fluorescence was measured using secondary antibody only. Mean fluorescence intensity was measured by flow cytometry. Data are summarized as the mean \pm SD and represent at least three replicates. (A) DR4; (B) DR5. Differences between two groups were determined by the Student's t-test. P>0.05 compared to CNE-1-LMP1 cells.

\section{Results}

Overexpression of LMP1 prevents TRAIL-induced apoptosis in NPC cells. Effect of TRAIL on cell survival in CNE-1 and CNE1-LMP1 cells was evaluated by using MTT cell viability assays. As shown in Fig. 1A, treatment with TRAIL caused cell death in CNE-1 cells in a dose- and timedependent manner but had little effect on the survival rate of CNE-1-LMP1 cells. Levels of apoptosis in CNE-1 and CNE-1-LMP1 cells treated with TRAIL were next measured by using Annexin V/PI staining and flow cytometry. Similarly, TRAIL induced significant apoptosis in CNE-1 cells in a time- and dose-dependent manner but caused minimal apoptosis in CNE-1-LMP1 cells (Fig. 1B). These results suggest that overexpression of LMP1 prevents TRAIL induction of apoptosis in NPC cells.

Expression of LMP1 does not affect expression of death receptors in NPC cell. Since TRAIL is known to trigger apoptosis via two types of death receptors, DR4 and DR5, expressions of these receptors were compared between CNE-1 and CNE-1-LMP1 cells. As shown in Fig. 2, cell surface expression levels of DR4 and DR5 were similar between these two cell lines $(\mathrm{P}>0.05)$. After treatment with TRAIL, cell surface expressions of DR4 and DR5 was slightly increased in both CNE-1 and CNE-1-LMP1 cell lines, but the increase 

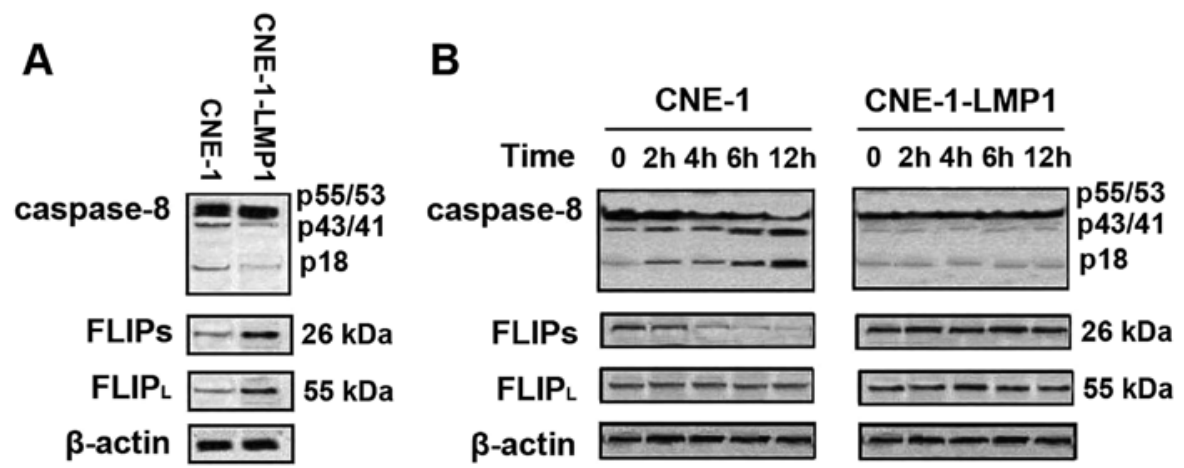

Figure 3. LMP1 overexpression maintained FLIP high expression upon TRAIL treatment. (A) Western blot analysis of FLIP and caspase-8 expression in CNE-1 and CNE-1-LMP1 cells. (B) Western blot analysis of FLIP and caspase-8 expression in CNE-1 and CNE-1-LMP1 cells after treated with $100 \mathrm{ng} / \mathrm{ml}$ of TRAIL for different periods of time.
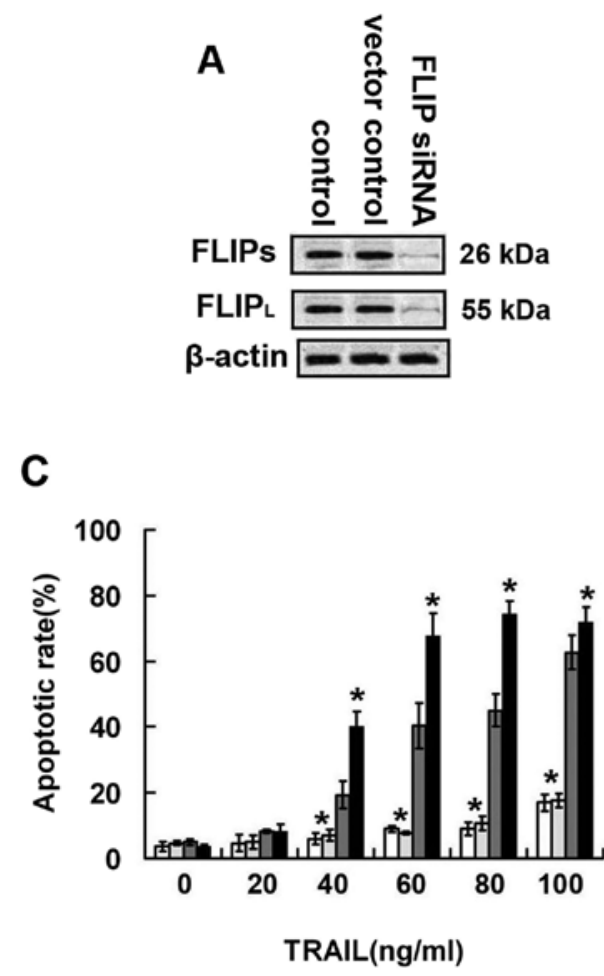
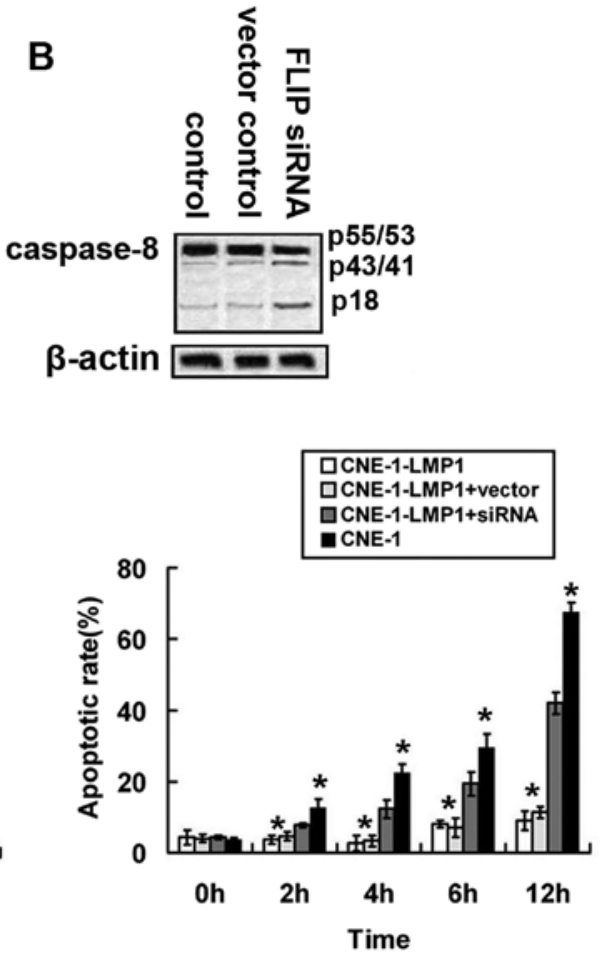

Figure 4. FLIP suppression mediates the effects of LMP1 in the decreased sensitivity of NPC cells to TRAIL. Western blot analysis of (A) FLIP and (B) caspase-8 expressions in the FLIP siRNA-transfected CNE-1-LMP1 cells. (C) Annexin V/PI assay analysis of apoptosis in the FLIP siRNA-transfected CNE-1-LMP1 cells after TRAIL treatment. The apoptotic rate was detected by flow cytometry analysis. Data are summarized as the mean \pm SD and represent three replicates. Differences between two groups were determined by the Student's t-test. "P<0.05 compared to FLIP siRNA-transfected CNE-1-LMP1 cells.

was again similar between the two cell lines (P>0.05) (Fig. 2). These results suggest that LMP1 does not affect expression of cell death receptors DR4 and DR5.

LMP1-induced resistance to TRAIL is associated with increased expression of FLIP. In order to determine the downstream molecular mechanism by which LMP1 induced resistance to TRAIL in NPC cells, we examined the effect of LMP1 on cleavage of caspase-8. It is established that cleavage of caspase- 8 is the most important event in extrinsic apoptotic pathway, and the recruitment of caspase- 8 into the DISC and the subsequent cleavage of it is inhibited by FLIP (20). There are two major isoforms of FLIP: long isoform $\left(\right.$ FLIP $\left._{L}\right)$ and short isoform $\left(\right.$ FLIP $\left._{S}\right)$. We next examined the effect of LMP1 on expressions of FLIP and caspase-8 cleavage. As shown in Fig. 3A, levels of FLIP proteins were increased while the cleavage of caspase- 8 was decreased in LMP1 expressing cells (CNE-1-LMP1) in comparison to CNE-1 cells without LMP1 overexpression. Treatment with TRAIL decreased expression of FLIP $_{S}$ in CNE-1 cells but not in CNE-1-LMP1 cells. Expression of FLIP $_{\mathrm{L}}$ was not affected by TRAIL in either cell line. Treatment with TRAIL stimulated caspase- 8 cleavage in a time-dependent manner in CNE-1 cells but not in CNE-1-LMP1 cells (Fig. 3B). Together, these results suggest that LMP1 effect on TRAIL function is associated with expression of FLIPs. 

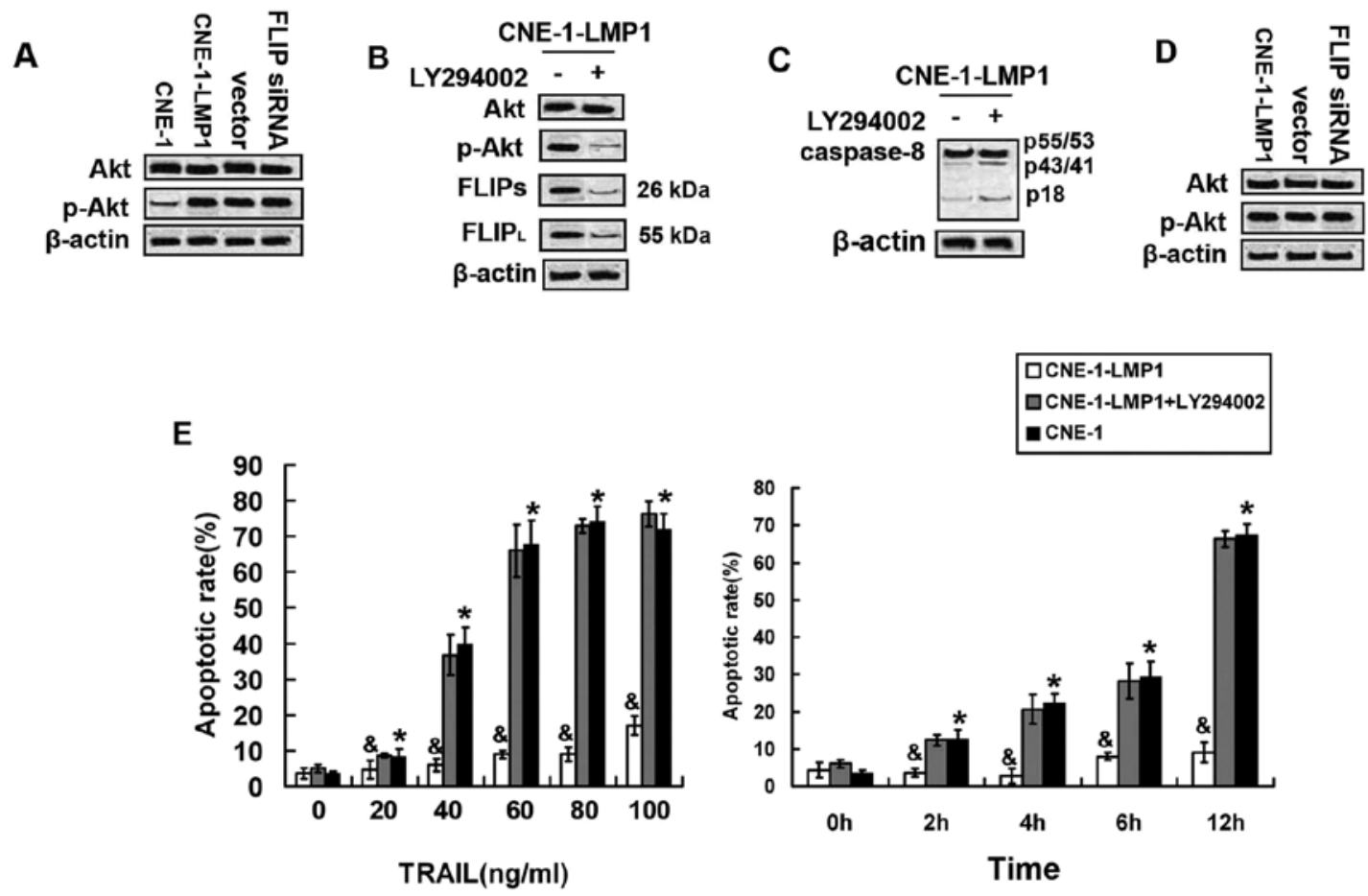

Figure 5. PI3-K inhibitor LY294002 inhibits FLIP expression and reverses LMP1-induced TRAIL resistance. (A) Western blot analysis of Akt and p-Akt expression in CNE-1, CNE-1-LMP1 and FLIP siRNA-transfected CNE-1-LMP1 cells. Western blot analysis of (A) Akt, p-Akt, FLIP and (B) caspase-8 expressions after treatment with $1 \mu \mathrm{M} \mathrm{LY} 294002$ for $8 \mathrm{~h}$. (C) Annexin V/PI assay analysis of apoptosis in the pretreated with $1 \mu \mathrm{M} \mathrm{LY} 294002$ for $8 \mathrm{~h}$, or not, NPC cells after TRAIL treatment. Cell apoptotic rate was detected by flow cytometry analysis. Data are summarized as mean \pm SD and represent three replicates. Differences between two groups were determined by the Student's t-test. "P>0.05 compared to CNE-1-LMP1 cells treated with LY294002, and P<0.05 compared to CNE-1-LMP1 cells treated with LY294002.

FLIP is involved in LMP1 induction of TRAIL resistance in NPC cells. To further determine the role of FLIP in LMP1 function, FLIP was knocked down by using siRNA, followed by evaluations of caspase- 8 cleavage and TRAIL induction of apoptosis. As shown in Fig. 4A and B, FLIP was efficiently knocked down by the specific siRNA, and the knockdown of FLIP led to increased cleavage of caspase- 8 and TRAILinduced apoptosis in CNE-1-LMP1. Upon treatment with TRAIL, the cell survival rate of CNE-1-LMP1 cells was decreased but still higher in CNE-1-LMP1 cells than in CNE-1 cells when FLIP was knocked down by siRNA $(\mathrm{P}<0.05)$. These results suggest that FLIP is an important factor of LMP1-indued TRAIL resistance, but no the only factor.

Expression of FLIP is PI3K/Akt-dependent in NPC cells. To investigate the mechanism of LPM1 induction of FLIP expression, we examined levels of Akt activation in CNE-1 and CNE-1-LMP1 cells. As shown in Fig. 5A, phosphorylation level of Akt was significantly higher in CNE-1-LMP1 cells than that in CNE-1 cells while levels of total Akt proteins were not different between the two cell lines. To determine the potential role of the elevated Akt activation in expression of FLIP, we treated the cells with PI3K inhibitor LY294002, followed by evaluation of FLIP expression, caspase-8 cleavage and TRAIL induction of apoptosis. As shown in Fig. 5B, expression of FLIP was decreased by inhibition of Akt activation with LY294002. In contrast, caspase-8 cleavage was enhanced by LY294002 (Fig. 5C). Importantly, inhibition of Akt by LY294002 brought the TRAIL-induced apoptosis in
CNE-1-LMP1 cells to the same level of that of CNE-1 cells (Fig. 5E) (P>0.05). The effect of LY294002 was eliminated when FLIP was knocked down (Fig. 5D). These results suggest that expression of FLIP is PI3K/Akt signaling pathway-dependent in NPC cells.

LMP1 does not affect the TRAIL-induced mitochondrial events. We subsequently investigated whether LMP1 regulated TRAIL-induced apoptosis by influencing mitochondrial membrane potential $(\Delta \Psi \mathrm{m})$ and cytochrome $\mathrm{c}$ release from mitochondria. The TRAIL-induced mitochondrial depolarization measured by $\mathrm{DiOC}_{6}(3)$ fluorescence was not different between CNE-1 and CNE-1-LMP1 cells (Fig. 6A). The level of $\Delta \Psi \mathrm{m}$ was not affected by TRAIL treatment in either CNE-1 or CNE-1LMP1 cell lines. As shown in Fig. 6B, cleavage of Bid was not affected by LMP1 overexpression or TRAIL treatment, either. Together, LMP1 and TRAIL do not affect mitochondrial events in NPC cells.

\section{Discussion}

Despite aggressive therapies, resistance to current treatment protocols is a major obstacle in clinical oncology. Most anticancer agents kill cancer cells by inducing apoptosis in cancer cells (21). TRAIL is a promising candidate of cancer therapy via induction of apoptosis but its potential application has been limited by the development of drug resistance. Some studies have shown that TRAIL can not induce apoptosis effectively in certain NPC cell lines (12). Therefore, defining the mecha- 

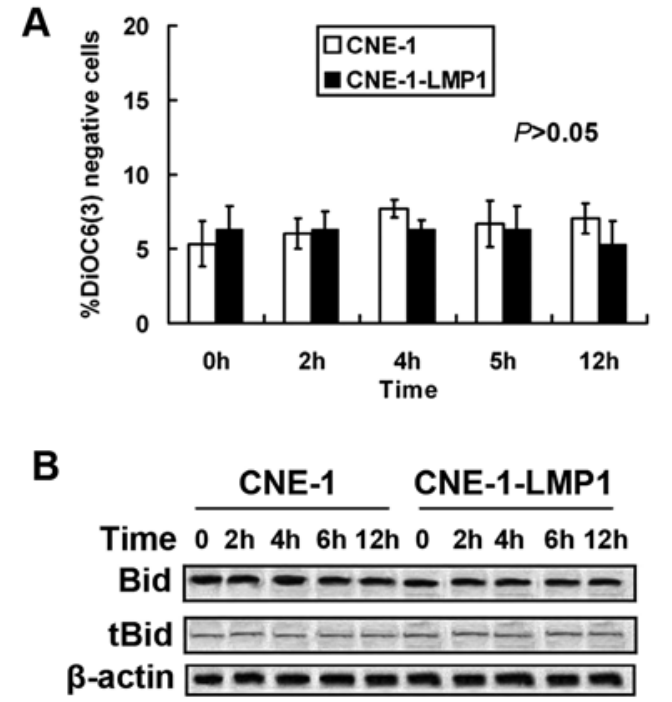

Figure 6. LMP1 could not affect TRAIL-induced mitochondrial events. (A) Mitochondrial depolarization as measure by DiOC6(3) fluorescence in CNE-1 and CNE-1-LMP1 cells after treated with $100 \mathrm{ng} / \mathrm{ml}$ of TRAIL for different periods of time. (B) Western blot analysis of Bid and truncated form of Bid expression in CNE-1 and CNE-1-LMP1 cells after treated with $100 \mathrm{ng} / \mathrm{ml}$ of TRAIL for different periods of time. Differences between two groups were determined by the Student's t-test. "P $>0.05$ compared to CNE1-LMP1 cells.

nisms of TRAIL resistance has become an important issue. In this study, we observed for the first time that LMP1 induces resistance to the TRAIL-mediated apoptosis and cell death in NPC cells.

Expression of EBV genes is present in almost all malignant NPC cells. These genes encode viral proteins that may contribute to malignant phenotypes (14). Several EBV genes are consistently expressed in NPC biopsies from humans. These genes include EBERs, EBNA1, LMP1, LMP2, BARF0 and BARF1 (22). Among them, LMP1 is capable of inducing malignant transformation in rodent fibroblasts (23) and has thus been considered as a viral oncogene. The presence of LMP1 in NPC tissue suggests its contribution to the EBV-mediated tumorigenesis (24). Silencing of the LMP1 gene by siRNA has been shown to induce cell cycle arrest and enhance chemosensitivity to bleomycin and cisplatin in EBV-positive nasopharyngeal carcinoma cells, suggesting a possible role for LMP1 in chemoresistance in NPC (16). No direct role has been reported for LMP1 in the development of TRAIL resistance. However, some reports indirectly imply a role for LMP1 in the development of TRAIL resistance in some malignant cells. For example, Snow et al (25) examined the sensitivity of EBV positive spontaneous lymphoblastoid cell lines derived from patients with post-transplant lymphoproliferative disease to soluble TRAIL. Despite abundant expression of death receptors, resistance to TRAIL-induced apoptosis was observed in all cell lines examined, suggesting a role for EBV that encodes LMP1 in the development of TRAIL resistance. Silencing of the LMP1 gene with siRNA in NPC cells has been shown to increase cleavage of caspase 3 and PARP and activation of Bax, implying that down-regulation of LMP1 may sensitize NPC cells to apoptosis (16). In this study, we tested the effect of LMP1 overexpression on TRAIL-induced apoptosis in NPC cells. First, we assessed the TRAIL sensitivity in NPC cell lines which expressed different level of LMP1. We found that CNE-1-LMP1 cells which expressed LMP1 stably were more resistant to TRAIL than CNE-1 which is an EBV-negative cell line. Our results show that LMP1 is important in regulating the sensitivity of NPC cells to TRAIL and can be a potential target for preventing or reversing development of TRAIL resistance in NPC.

Both NPC cell lines examined in this study did not show Bid cleavage and dissipation of $\Delta \Psi \mathrm{m}$ in response to TRAIL treatment, suggesting that TRAIL induces NPC cell apoptosis in a mitochondrion-independent manner and LMP1 may affect TRAIL sensitivity by inhibiting the extrinsic apoptotic pathway. FLIP has homology to caspase- 8 and -10 , but lacks protease activity (26). Therefore, it is thought that FLIP recruitment to the DISC in the place of the caspases blocks their activation to inhibit the extrinsic apoptotic pathway. Some overexpression studies have suggested that FLIP is an inhibitor of caspase-8 activation and TRAIL-induced apoptosis (27). While FLIP $_{S}$ inhibits apoptosis by preventing the recruitment of caspase- 8 into the DISC, the role of FLIP $_{\mathrm{L}}$ is controversial. Indeed, FLIP $_{\mathrm{L}}$ has been reported as both an inhibitor and an inducer of apoptosis, possibly depending on its expression level (28). In this study, results show an important role for FLIP in LMP1-mediated TRAIL resistance but FLIP may be responsible for only a part of the effect of LMP1 in TRAIL resistance in NPC cells.

To date, many factors that can alter the sensitivity of cancer cells to TRAIL have been identified. However, the underlying molecular mechanisms remain to be defined. For example, overexpression of anti-apoptotic molecules and activation of pro-survival signal pathway may also contribute to the insensitivity to TRAIL. The PI3-K/Akt-dependent pathway is the primary pro-survival signal pathway (29). It has been shown that modulation of Akt activity by combining pharmacological drugs with genetic alteration of Akt expression induces cellular responsiveness to TRAIL (30). In this study, Akt activation in TRAIL-resistant CNE-1-LMP1 cells was much higher than that in TRAIL sensitive CNE-1 cells, implying a role for Akt activation in regulation of TRAIL sensitivity and in effect of LMP1 in TRAIL resistance in NPC. This is consistent with some previous studies that LMP1 activates the PI3-K/Akt-dependent pathway to promote cell survival (17). More studies have shown that silencing the LMP1 gene can enhance chemosensitivity while inhibiting the PI3-K/ Akt signaling pathway in the EBV-positive nasopharyngeal carcinoma cells (16). Here we show that inhibition of PI3K/ Akt pathway suppresses expression of FLIP and reverses the LMP1-induced TRAIL resistance, supporting the notion that LMP1 reduces TRAIL sensitivity in NPC cells by activating the PI3K/Akt-dependent signaling pathway. Since silencing the FLIP gene does not affect the PI3K/Akt signaling pathway, up-regulation of FLIP expression is downstream of Akt in the LMP1-induced TRAIL resistance.

In summary, results from this study show that the LMP1induced insensitivity of NPC cells to TRAIL is mediated by the PI3K/Akt-dependent signaling pathway. Inhibition of the PI3K/Akt-dependent pathway is sufficient to suppress FLIP expression and reverse TRAIL resistance. Thus, results from this study provide a new target to treat NPC. 


\section{Acknowledgements}

We thank Dr Wenhong Cao of Division of Translational Biology, the Hamner Institutes for Health Sciences, Research Triangle Park, USA for assistance in preparing this manuscript.

\section{References}

1. Walczak H and Krammer PH: The CD95 (APO-1/Fas) and the TRAIL (APO-2L) apoptosis systems. Exp Cell Res 256: 58-66, 2000.

2. Pan G, Ni J, Wei YF, Yu G, Gentz R and Dixit VM: An antagonist decoy receptor and a death domain-containing receptor for TRAIL. Science 277: 815-818, 1997.

3. Green DR and Evan GI: A matter of life and death. Cancer Cell 1: $19-30,2002$.

4. Luo X, Budihardjo I, Zou H, Slaughter C and Wang X: Bid, a $\mathrm{Bcl}-2$ interacting protein, mediates cytochrome $\mathrm{c}$ release from mitochondria in response to activation of cell surface death receptors. Cell 94: 481-490, 1998.

5. Siddiqui IA, Malik A, Adhami VM, Asim M, Hafeez BB, Sarfaraz S and Mukhtar H: Green tea polyphenol EGCG sensitizes human prostate carcinoma LNCaP cells to TRAIL-mediated apoptosis and synergistically inhibits biomarkers associated with angiogenesis and metastasis. Oncogene 27: 2055-2063, 2008.

6. Szliszka E, Zydowicz G, Janoszka B, Dobosz C, KowalczykZiomek $\mathrm{G}$ and Krol W: Ethanolic extract of Brazilian green propolis sensitizes prostate cancer cells to TRAIL-induced apoptosis. Int J Oncol 38: 4941-4953, 2011.

7. Tang L, Mao Y, Liu L, Liang S, Chen Y, Sun Y, Liao X, Lin A, Liu M, Li L and Ma J: The volume to be irradiated during selective neck irradiation in nasopharyngeal carcinoma: analysis of the spread patterns in lymph nodes by magnetic resonance imaging. Cancer 115: 680-688, 2009.

8. Isobe $\mathrm{K}$, Ito $\mathrm{H}$, Shigematsu $\mathrm{N}$, Kawada T, Yasuda $\mathrm{S}$, Hara $\mathrm{R}$ Machida N, Takano H, Uchida Y, Uno T, Kitahara H and Kubo A: Advanced nasopharyngeal carcinoma treated with chemotherapy and radiotherapy: distant metastasis and local recurrence. Int J Oncol 12: 1183-1187, 1998.

9. Shao JY, Wang HY, Huang XM, Feng QS, Huang P, Feng BJ, Huang LX, Yu XJ, Li JT, Hu LF, Ernberg I and Zeng YX: Genome-wide allelotype analysis of sporadic primary nasopharyngeal carcinoma from southern China. Int J Oncol 17: 1267-1275, 2000.

10. Teng MS, Brandwein Gensler MS, Teixeira MS, Martignetti JA and Duffey DC: A study of TRAIL receptors in squamous cell carcinoma of the head and neck. Arch Otolaryngol Head Neck Surg 131: 407-412, 2005.

11. Chen LC, Chung IC, Hsueh C, Tsang NM, Chi LM, Liang Y, Chen CC, Wang LJ and Chang YS: The antiapoptotic protein, FLIP, is regulated by heterogeneous nuclear ribonucleoprotein $\mathrm{K}$ and correlates with poor overall survival of nasopharyngeal carcinoma patients. Cell Death Differ 17: 1463-1473, 2010.

12. Ozoren N, Fisher MJ and Kim K: Homozygous deletion of the death receptor DR4 gene in a nasopharyngeal cancer cell line is associated with TRAIL resistance. Int J Oncol 16: 917-925, 2000.

13. Kijima T, Kinukawa N, Gooding WE and Uno M: Association of Epstein-Barr virus with tumor cell proliferation: clinical implication in nasopharyngeal carcinoma. Int J Oncol 18: 479-485, 2001.

14. Tang YL, Lu JH, Cao L, Wu MH, Peng SP, Zhou HD, Huang C, Yang YX, Zhou YH, Chen Q, Li XL, Zhou M and Li GY: Genetic variations of EBV-LMP1 from nasopharyngeal carcinoma biopsies: potential loss of T cell epitopes. Braz J Med Biol Res 41: $110-116,2008$
15. Du CX, Wen BG, Li DR, Peng X, Hong CQ, Chen JY, Lin ZZ, Hong X, Lin YC, Xie LX, Wu MY and Zhang H: Arsenic trioxide reduces the invasive and metastatic properties of nasopharyngeal carcinoma cells in vitro. Braz J Med Biol Res 39: 677-685, 2006.

16. Mei YP, Zhou JM, Wang Y, Huang H, Deng R, Feng GK, Zeng YX and Zhu XF: Silencing of LMP1 induces cell cycle arrest and enhances chemosensitivity through inhibition of AKT signaling pathway in EBV-positive nasopharyngeal carcinoma cells. Cell Cycle 6: 1379-1385, 2007.

17. Dawson CW, Tramountanis G, Eliopoulos AG and Young LS Epstein-Barr virus latent membrane protein 1 (LMP1) activates the phosphatidylinositol 3-kinase/Akt pathway to promote cell survival and induce actin filament remodeling. J Biol Chem 278: 3694-7304, 2003

18. Oka N, Tanimoto $S$, Taue R, Nakatsuji H, Kishimoto T, Izaki H, Fukumori T, Takahashi M, Nishitani M and Kanayama H: Role of phosphatidylinositol-3 kinase/Akt pathwayin bladder cancer cell apoptosis induced by tumor necrosis factor-related apoptosisinducing ligand. Cancer Sci 10: 1093-1098, 2006.

19. Dieterle A, Orth R, Daubrawa M, Grotemeier A, Alers S, Ullrich S, Lammers R, Wesselborg S and Stork B: The Akt inhibitor triciribine sensitizes prostate carcinoma cells to TRAIL-induced apoptosis. Int J Cancer 125: 932-941, 2009.

20. Sarkar MC, Bae SI, Reu FJ, Jacobs BS, Lindner DJ and Borden EC: Down-regulation of Bcl-2, FLIP or IAPs (XIAP and survivin) by siRNAs sensitizes resistant melanoma cells to Apo2L/TRAILinduced apoptosis. Cell Death Differ 11: 915-923, 2004.

21. Kaufmann SH and Earnshaw WC: Induction of apoptosis by cancer chemotherapy. Exp Cell Res 256: 42-49, 2000.

22. Hitt MM, Allday MJ and Hara T: EBV gene expression in an NPC-related tumor. EMBO J 8: 2639-2651, 1989.

23. Wang D, Liebowitz D and Kieff E: An EBV membrane protein expressed in immortalized lymphocytes transforms established rodent cells. Cell 43: 831-840, 1985.

24. Lin SY, Tsang NM and Kao SC: Presence of Epstein-Barr virus latent membrane protein 1 gene in the nasopharyngeal swabs from patients with nasopharyngeal carcinoma. Head Neck 23: 194-200, 2001.

25. Snow AL, Vaysberg M, Krams SM and Martinez OM: $\mathrm{EBV}^{+} \mathrm{B}$ lymphoma cell lines from patients with post-transplant lymphoproliferative disease are resistant to TRAIL-induced apoptosis. Am J Transplant 6: 976-985, 2006.

26. Tschopp J, Irmler $M$ and Thome M: Inhibition of fas death signals by FLIPs. Curr Opin Immunol 10: 552-558, 1998.

27. Wang J, Lobito AA, Shen F, Hornung F, Winoto A and Lenardo MJ: Inhibition of Fas-mediated apoptosis by the $\mathrm{B}$ cell antigen receptor through c-FLIP. Eur J Immunol 30: 155-163, 2000.

28. Chang DW, Xing Z, Pan Y, Algeciras-Schimnich A, Barnhart BC Yaish-Ohad S, Peter ME and Yang X: c-FLIP(L) is a dual function regulator for caspase-8 activation and CD95-mediated apoptosis. EMBO J 21: 3704-3714, 2002.

29. Liu HY, Wen GB, Han JM, Hong T, Zhuo DG, Liu ZQ and Cao WH: Inhibition of gluconeogenesis in primary hepatocytes by stromal cell-derived factor-1 (SDF-1) through a c-Src/ Akt-dependent signaling pathway. J Biol Chem 283: 3064230649, 2008

30. Oka N, Tanimoto S, Taue R, Nakatsuji H, Kishimoto T, Izaki H, Fukumori T, Takahashi M, Nishitani $M$ and Kanayama HO: Role of phosphatidylinositol-3 kinase/Akt pathway in bladder cancer cell apoptosis induced by tumor necrosis factor-related apoptosis-inducing ligand. Cancer Sci 97: 1093-1098, 2006. 